\title{
COMPARISON OF LONG-PERIOD SURFACE WAVE AMPLITUDE AND PHASE ANOMALIES FOR TWO MODELS OF GLOBAL LATERAL HETEROGENEITY
}

\author{
Susan Y. Schwartz and Thorne Lay
}

Department of Geological Sciences, The University of Michigan

\begin{abstract}
Surface wave raytracing calculations for periods of 150 to $250 \mathrm{~s}$ were performed using the two models of global phase velocity heterogeneity produced by Harvard and CIT, providing a comparison of predicted amplitude and phase anomalies. Theoretical $R_{3} / R_{2}$ and $G_{3} / G_{2}$ amplitude asymmetries resulting from focussing and defocussing have similar azimuthal distributions of the more pronounced anomalies for the two structures. The azimuthal patterns of long-period Rayleigh and Love wave phase anomalies about a given source region also resemble one another for the two models. Travel time errors as large as 12 $s$ are accumulated for $200 \mathrm{~s}$ period $R_{3}$ arrivals by neglecting the raypath deviations from great circles. Such errors may be significant when inverting for earth structure or when applying corrections for lateral heterogeneity in source studies.
\end{abstract}

\section{Introduction}

Long-period surface wave amplitude asymmetries between odd and even orbit surface wave arrivals at a given station have been reported for many earthquakes (e.g., Niazi and Kanamori, 1981; Lay and Kanamori, 1985). These have been interpreted as geometric effects due to lateral heterogeneity of the mantle (Wong and Woodhouse, 1983; Lay and Kanamori, 1985). Horizontal gradients in phase velocity cause surface waves to propagate along paths that deviate from great circles, resulting in focussing and defocussing of amplitudes. Accounting for the effects of lateral heterogeneity on both the amplitude and phase spectra of surface waves is important for accurate determination of source parameters and attenuation. The use of regionalized phase velocities to correct long-period surface wave phase spectra for propagation effects prior to source mechanism inversions has improved the agreement between observations and models (Nakanishi and Kanamori, 1982). The potential to incorporate amplitude corrections in these inversions exists; however, because surface wave amplitudes in laterally heterogeneous media are sensitive to the second spatial derivative of velocity transverse to the raypath (Wong and Woodhouse, 1983), small variations between velocity models may predict very different amplitude corrections. Two models of global lateral heterogeneity of the upper mantle have recently been proposed. The purpose of this paper is to quantitatively compare the amplitude and phase anomalies predicted by both models in order to assess their similarity and to evaluate their potential use in the improvement of source and attenuation determinations.

\section{Copyright 1985 by the American Geophysical Union.}

Paper number $5 \mathrm{~L} 6448$.

0094-8276/85/005L-6448\$03.00

\section{Method}

Surface wave raytracing calculations were performed using the long-period Rayleigh and Love wave spherical harmonic phase velocity distributions of Nakanishi and Anderson (1984) (CIT) and Woodhouse and Dziewonski (1984) (Harvard). Following Lay and Kanamori (1985), the eikonal equations in spherical coordinates were numerically integrated to calculate the raypaths and intensities of rays leaving the source region in $1^{\circ}$ azimuthal increments. The low order spherical harmonic expansions $(l=6$ for CIT and $l=8$ for Harvard) should be heavily smoothed representations of the actual heterogeneity, but the velocity models vary slowly enough to satisfy the conditions necessary to apply ray theory. Amplitude and phase anomalies were calculated at receivers at representative distances of $60^{\circ}$ and $120^{\circ}$ from three source regions for both $R_{2}$ and $R_{3}$ arrivals. The amplitude anomaly at a given position was computed by summing the number of rays passing within one wavelength of that point in the heterogeneous earth as the wavefront sweeps across it and normalizing by the corresponding sum for the homogeneous earth. Ray density was used in the amplitude determinations because the integrated ray intensity calculations are unstable near caustics, which are frequently encountered. The phase, or travel time anomaly at a given position is defined by the difference in travel time for a ray propagating from the source to that point through the heterogeneous structure compared to the travel time of a ray traveling through a homogenous reference structure (along the great circle) to that point.

Lay and Kanamori (1985) demonstrated the sensitivity of surface wave raypaths to the distribution of lateral heterogeneity around different source regions using the CIT model. They also demonstrated the period dependence of the ray calculations resulting from variations in the phase velocity heterogeneity for different period surface waves, as well as the differences expected for Love and Rayleigh waves of the same period. Consequently source regions in Japan, California and Iran were selected to compare amplitude and phase anomalies predicted by the CIT and Harvard models for periods of $150 \mathrm{~s}, 200 \mathrm{~s}$, and $250 \mathrm{~s}$ for both Love and Rayleigh waves. In general, as the range in phase velocity variation increases, the ray deflections become more pronounced and resulting amplitude and phase anomalies are magnified. Thus, a larger range in anomalies is expected for shorter period signals than for longer periods as well as for Love waves compared with Rayleigh waves of the same period.

\section{Results}

The global $200 \mathrm{~s}$ period Rayleigh wave phase velocity variations, centered on Japan, for the Harvard and CIT models are shown in Figure 1 , along with $R_{2}$ and $R_{3}$ raypaths for each $1^{\circ}$ 


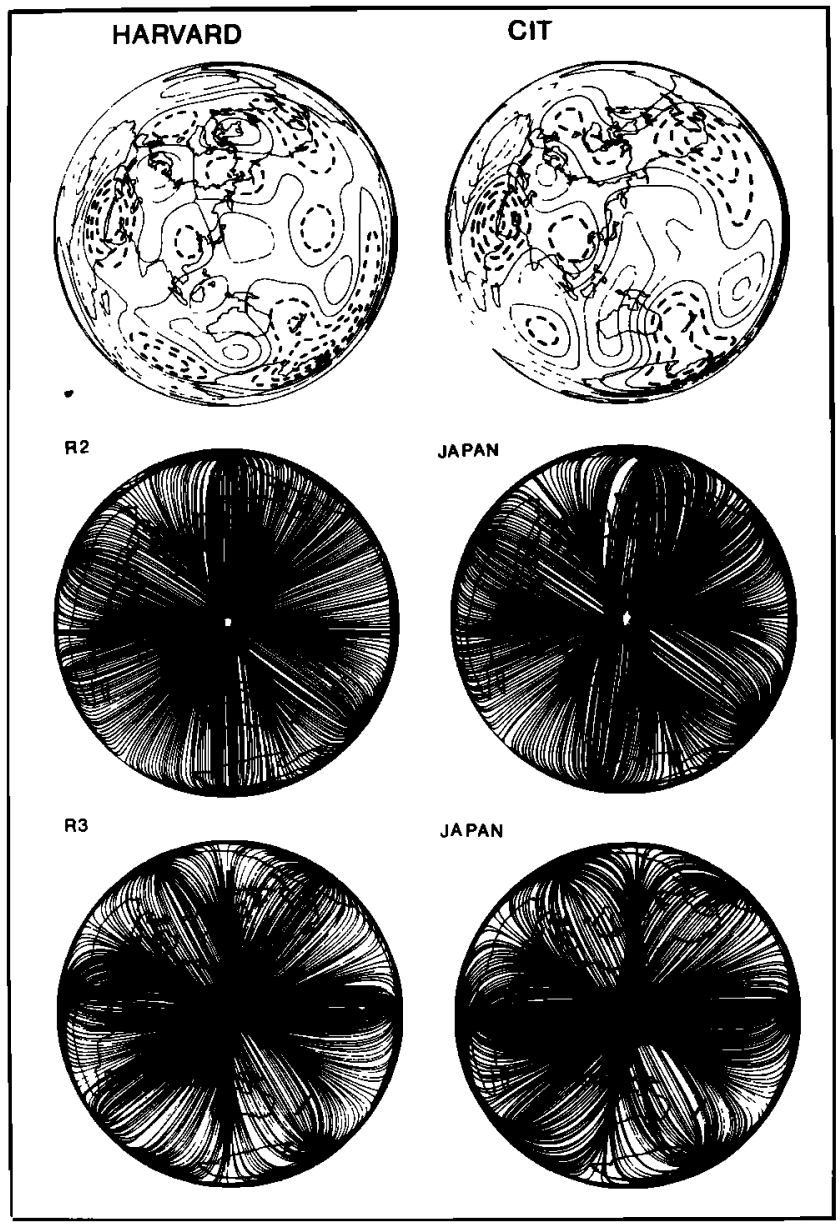

Figure 1. $200 \mathrm{~s}$ period Rayleigh wave phase velocity distributions for the Harvard and CIT models and corresponding $\mathrm{R}_{2}$ and $R_{3}$ raypaths for a source in Japan. The contour interval is $0.02 \mathrm{~km} / \mathrm{s}$, with dashed contours indicating slower than average velocities.

take off azimuth increment from a source in Japan. The maps are equal area projections, with the antipode distributed around the circumference. The $R_{2}$ raypaths are incoming rays which travel $180^{\circ}$ from the antipode to the source region, and the $R_{3}$ raypaths are outgoing rays traveling the next $180^{\circ}$ from the source region back to the antipode. For a homogeneous model, the raypaths would be straight radial spokes meeting at the center of each projection. The differences in the velocity models give rise to different patterns in the $R_{2}$ and $R_{3}$ raypaths. The largest difference between the models in $R_{2}$ raypaths occurs at azimuths to the north and northeast of Japan, while the greatest difference for the $R_{3}$ raypaths occurs at azimuths to the south and southwest. Numerous other examples of such raypath calculations are presented by Lay and Kanamori (1985).

In Figure 1 the $200 \mathrm{~s}$ phase wavefront at any moment in time would be a slightly irregular concentric ring centered on the Japan source region. Positions along this wavefront that have an increased ray density would have larger amplitude signals, while areas with deficient ray density would have low amplitudes. While it is possible to directly compare $R_{2}$ or $R_{3}$ amplitude predictions for the two structures, we choose to compare the amplitude ratios $R_{3} / R_{2}$ because in actual data asymmetries in these ratios are readily apparent and generally not produced by source processes or attenuation.

Calculated $R_{3} / R_{2}$ amplitude asymmetries produced by focussing and defocussing at receivers $60^{\circ}$ and $120^{\circ}$ from the three source regions show similar azimuthal distributions for both the Harvard and CIT velocity models. This reflects the similarity of the long wavelength components in the models. The predicted amplitude ratio patterns for $200 \mathrm{~s}$ period Rayleigh waves from Japan and North America are shown in Figure 2. For a homogeneous earth these ratios would have a constant value of unity at all azimuths. Although there are large local disparities in predicted amplitudes, the overall patterns are quite similar. The amplitude asymmetries are as large as a factor of 2 for Japan, and are noticeably smaller for North America and Iran (not shown). The magnitude of the asymmetry increases to a factor of 2.5 for $150 \mathrm{~s}$ period signals, and decreases to 1.5 for 250 $\mathrm{s}$ period signals, but otherwise the azimuthal patterns are similar to those in Figure 2. Similar calculations of $\mathrm{G}_{3} / \mathrm{G}_{2}$ ratios confirm that Love waves show larger asymmetries than Rayleigh waves at the same period and have quite different azimuthal variations. The CIT phase velocity distributions predict slightly larger amplitude asymmetries than the Harvard models for sources in both Japan and North America (Figure 2), which is

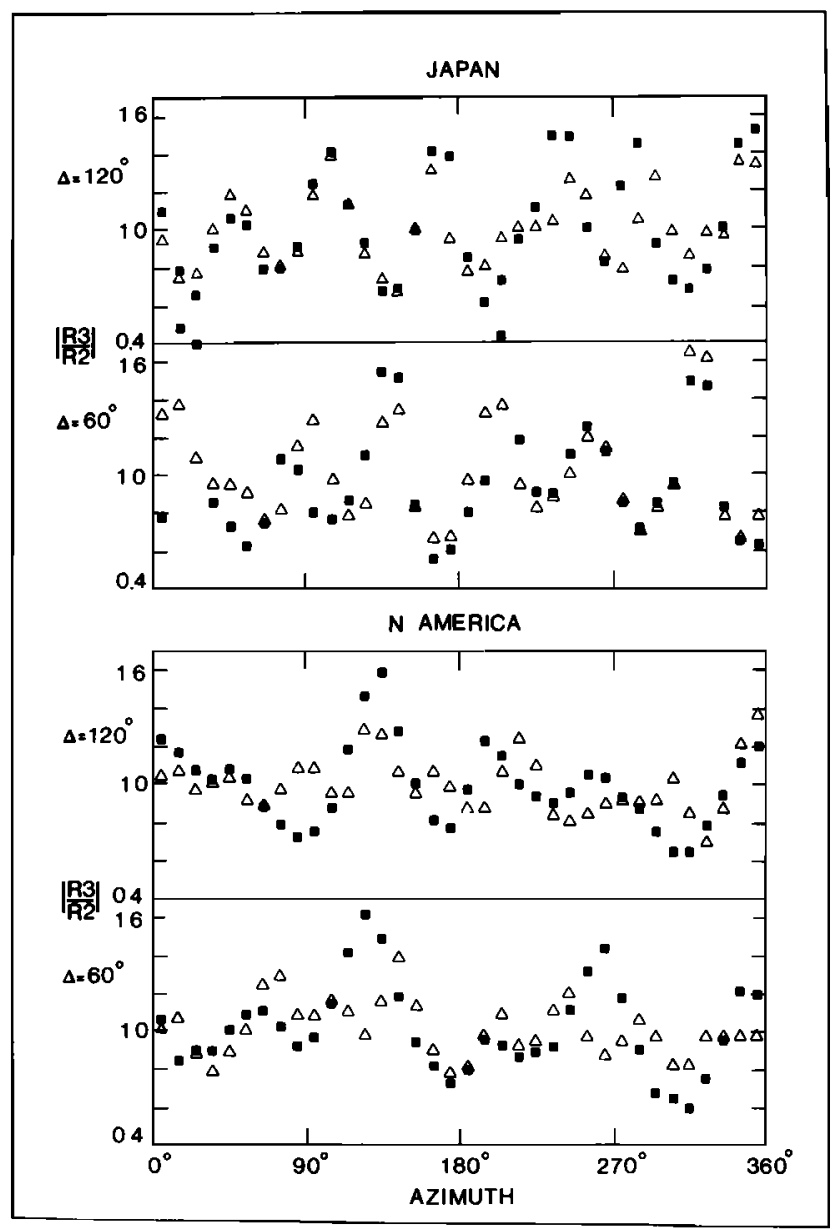

Figure 2. Azimuthal variation of predicted $R_{3} / R_{2}$ amplitude ratios for $200 \mathrm{~s}$ period Rayleigh waves for the Harvard structure (open triangles) and the CIT structure (solid squares). 
a result of the greater range in phase velocity variation in the former model.

Amplitude asymmetries predicted by the Harvard model at receivers $60^{\circ}$ and $120^{\circ}$ from different source regions were correlated with corresponding predictions for the CIT model. Correlation coefficients and slopes of best-fit lines for these amplitude asymmetry comparisons are listed for different periods and source regions in Table 1 . Slopes greater than unity for all but one of the Rayleigh wave cases show that the CIT model consistently predicts larger amplitude asymmetries than the Harvard model. In all but two cases the correlation of the two models is significant at the $95 \%$ confidence level, and the correlation is significant at the $98 \%$ confidence level for all calculations for the Japanese source region. These correlations change little with distance from the source or with period, and in general Love waves show less agreement between predicted amplitudes for the two structures.

The azimuthal pattems of Rayleigh wave phase anomalies about a given source region show very similar long wavelength trends for the two velocity models (Figure 3). The Japanese source region produces the largest range in phase anomalies,

Table 1. Correlation of Predicted Amplitude and Phase Anom. for the Harvard and CIT Velocity Structures

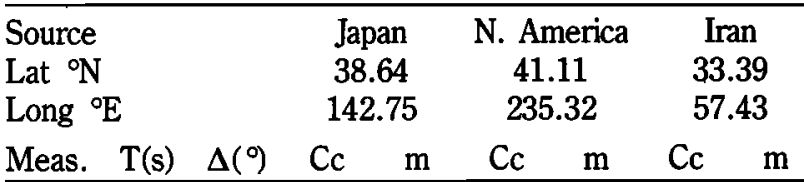

Amplitude Anomalies

\begin{tabular}{lrrrrrrcr}
\hline $\mathrm{R}_{3} / \mathrm{R}_{2}$ & 150 & 60 & .67 & 1.84 & .28 & 3.05 & .42 & 1.85 \\
$\mathrm{R}_{3} / \mathrm{R}_{2}$ & 150 & 120 & .65 & 1.35 & .13 & 10.09 & .49 & 1.90 \\
$\mathrm{R}_{3} / \mathrm{R}_{2}$ & 200 & 60 & .69 & 1.46 & .35 & 3.90 & .39 & 1.56 \\
$\mathrm{R}_{3} / \mathrm{R}_{2}$ & 200 & 120 & .68 & 1.57 & .43 & 2.88 & .42 & 1.06 \\
$\mathrm{G}_{3} / \mathrm{G}_{2}$ & 200 & 60 & .55 & 0.78 & .39 & 0.45 & -.035 & -.05 \\
$\mathrm{G}_{3} / \mathrm{G}_{2}$ & 200 & 120 & .41 & 0.70 & .41 & 0.72 & .12 & 0.34 \\
$\mathrm{R}_{3} / \mathrm{R}_{2}$ & 250 & 60 & .58 & 1.73 & .60 & 1.64 & .47 & 1.15 \\
$\mathrm{R}_{3} / \mathrm{R}_{2}$ & 250 & 120 & .54 & 2.03 & .54 & 2.06 & .53 & 0.67 \\
Travel & Time & Anomalies & & & & & \\
$\mathrm{R}_{2}$ & 150 & 60 & .80 & 2.61 & .86 & 1.81 & .68 & 0.70 \\
$\mathrm{R}_{2}$ & 150 & 120 & .71 & 2.52 & .76 & 1.61 & .81 & 1.30 \\
$\mathrm{R}_{3}$ & 150 & 60 & .54 & 3.42 & .91 & 1.99 & .68 & 2.93 \\
$\mathrm{R}_{3}$ & 150 & 120 & .62 & 3.24 & .88 & 1.79 & .73 & 2.79 \\
$\mathrm{R}_{2}$ & 200 & 60 & .88 & 1.89 & .92 & 1.59 & .84 & 1.46 \\
$\mathrm{R}_{2}$ & 200 & 120 & .70 & 2.72 & .82 & 1.46 & .76 & 0.84 \\
$\mathrm{R}_{3}$ & 200 & 60 & .79 & 2.52 & .93 & 1.71 & .73 & 1.58 \\
$\mathrm{R}_{3}$ & 200 & 120 & .84 & 2.28 & .90 & 1.56 & .70 & 1.55 \\
$\mathrm{G}_{2}$ & 200 & 60 & .83 & 1.13 & .78 & 1.33 & .28 & 0.63 \\
$\mathrm{G}_{2}$ & 200 & 120 & .67 & 1.06 & .59 & 1.38 & .35 & 0.51 \\
$\mathrm{G}_{3}$ & 200 & 60 & .53 & 0.74 & .84 & 1.25 & .07 & 14.01 \\
$\mathrm{G}_{3}$ & 200 & 120 & .56 & $\mathbf{0 . 8 7}$ & .89 & 1.13 & .037 & 9.72 \\
$\mathrm{R}_{2}$ & 250 & 60 & .87 & 2.09 & .93 & 1.46 & .61 & 1.03 \\
$\mathrm{R}_{2}$ & 250 & 120 & .79 & 2.42 & .85 & 1.40 & .50 & 1.11 \\
$\mathrm{R}_{3}$ & 250 & 60 & .92 & 2.35 & .93 & 1.75 & .35 & 0.49 \\
$\mathrm{R}_{3}$ & 250 & 120 & .90 & 2.19 & .94 & 1.59 & .57 & 0.74 \\
\hline
\end{tabular}

$\mathrm{C}_{\mathrm{c}}$ is the correlation coefficient and $\mathrm{m}$ is the slope of the bestfit line through the amplitude and travel time anomaly comparisons. $\mathrm{N}=36$; $\mathrm{Cc} \geq 0.50$ indicates a correlation significant at the $99 \%$ confidence level, while $\mathrm{C} c \geq 0.40$ indicates a correlation significant at the $98 \%$ confidence level.

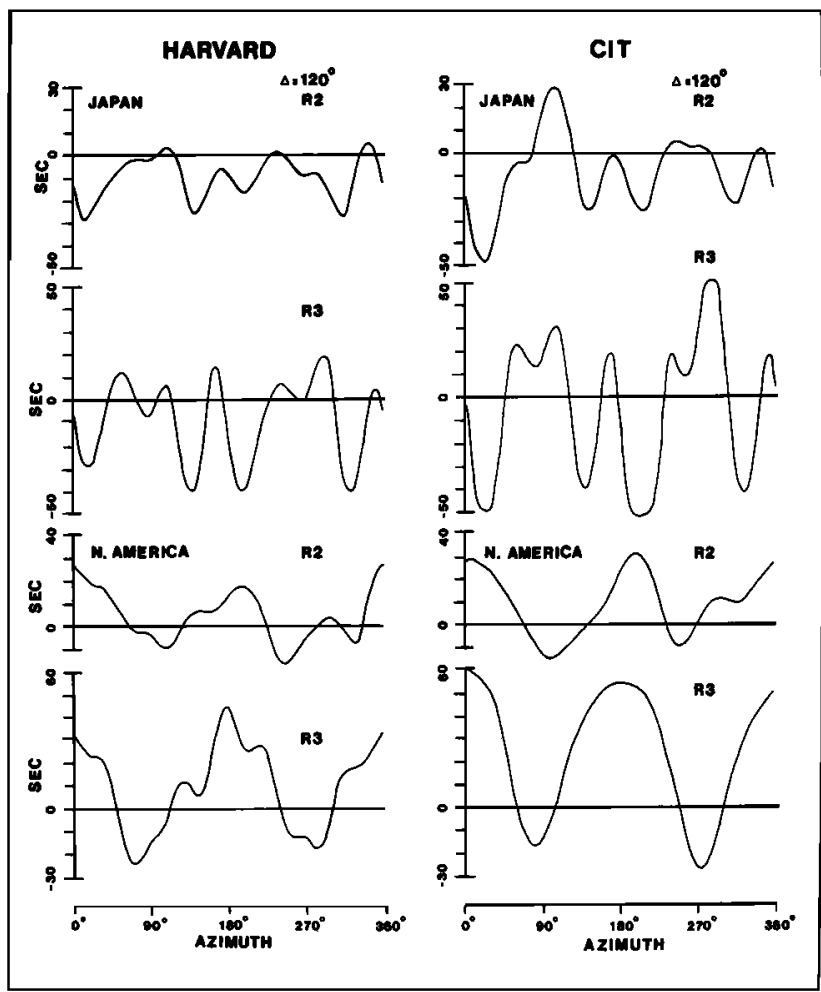

Figure 3. Azimuthal variation of predicted travel time anomalies relative to a homogeneous earth for the Harvard and CIT models of lateral heterogeneity. The calculations are for 200 $s$ period $R_{2}$ and $R_{3}$ arrivals at receivers $120^{\circ}$ from sources in Japan and North America.

with the CIT structure predicting travel time variations relative to a homogeneous earth as large as $75 \mathrm{~s}$ for $R_{2}$ and $100 \mathrm{~s}$ for $R_{3}$ for $200 \mathrm{~s}$ period arrivals. Longer propagation paths accumulate larger travel time anomalies.

Table 1 gives statistics for the predicted phase anomalies calculated in a similar manner to the amplitude comparisons. In most instances, the slope of the best-fit line through the phase anomaly comparison is greater than one, demonstrating the enhanced effect of the CIT structure. The correlation of phase anomalies between the models is significant at the $99 \%$ confidence level for all Rayleigh wave arrivals with one exception (Table 1). Love wave phase anomalies show less correlation than Rayleigh waves; however, this correlation is still significant at the $99 \%$ confidence level for waves from sources in Japan and North America.

The assumption that surface wave energy propagates along great circle paths is implicit in the derivation of both models of global lateral heterogeneity. Because the phase velocity variations of the Harvard and CIT models are large enough to cause substantial deflections in the surface wave raypaths, we have computed the error in travel times due to neglecting the raypath deviations for these models. The average phase velocity along the great circle to a point on an actual raypath was obtained by integration through the heterogeneous structure. This is the standard procedure used to obtain propagation corrections in source studies. The difference in travel time along the actual ray from that along the artificial great circle raypath is defined to be a great circle anomaly. The azimuthal patterns 


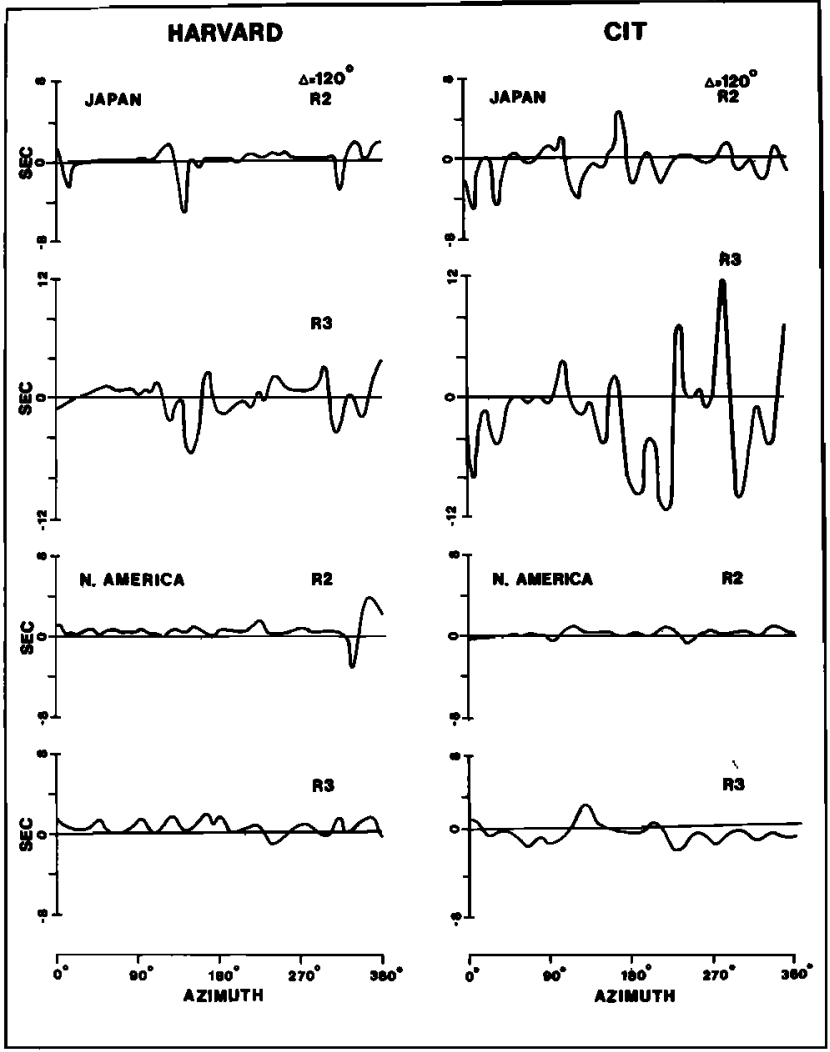

Figure 4. Azimuthal variation of predicted great circle anomalies for the Harvard and CIT velocity structures.

of the resulting great circle travel time anomalies for the two models are shown in Figure 4 for $200 \mathrm{~s}$ period $\mathrm{R}_{2}$ and $\mathrm{R}_{3}$ arrivals at receivers $120^{\circ}$ from Japan and North America. Neglecting the raypath deviations from the great circle leads to as much as a $\pm 12 \mathrm{~s}$ error in the calculated travel times for $R_{3}$ arrivals from Japan using the CIT structure, while the Harvard structure yields travel time anomalies only half as large. This results from the greater raypath deflections produced by the larger velocity variations in the CIT structure. The North American and Iranian source regions yield almost flat anomaly patterns, indicating that raypaths from these source regions do not significantly violate the great circle assumption for $200 \mathrm{~s}$ period Rayleigh waves. Although a travel time anomaly of $12 \mathrm{~s}$ accumulated over a path length of $480^{\circ}$ is usually negligible, the greater variation in phase velocities at shorter periods may require actual raypath calculations when inverting shorter period surface waves for mantle heterogeneity, or when using existing earth models to compute phase corrections to apply in source studies.

Attempts have been made to use both global and regional models of lateral heterogeneity to predict surface wave amplitudes observed at various stations (Lay and Kanamori, 1985; Yomogida and Aki, 1984). While these attempts have been successful in establishing the relationship between lateral heterogeneity and surface wave amplitude anomalies, they have been less successful in actually matching observed anomalies. This is not surprising given the variation in predicted amplitude anomalies found above for the two existing models of lateral heterogeneity. These models are preliminary attempts to define the phase velocity variations in the mantle, and although their long wavelength features are very similar, their local differences can be quite significant. It seems that even for very long periods the existing models do not yet provide sufficiently reliable amplitude corrections for inclusion in source inversion studies. The calculations in this paper are encouraging in that the predicted behavior for the two models is not dramatically different. This suggests that significant progress has been made in actually resolving true earth heterogeneity.

Acknowledgments. We thank I. Nakanishi and J. Woodhouse for providing us with their spherical harmonic expansions. We also appreciate comments on the manuscript made by C. Lynnes.

\section{References}

Lay, T., and H. Kanamori, Geometric effects of global lateral heterogeneity on long-period surface wave propagation, $J$. Geophys. Res., 90, 605-621, 1985.

Nakanishi, I., and H. Kanamori, Effect of lateral heterogeneity and source process time on the linear moment tensor inversion of long-period Rayleigh waves, Bull. Seismol. Soc. Amer., 72, 2063-2080, 1982.

Nakanishi, I., and D. L. Anderson, Measurements of mantle wave velocities and inversion for lateral heterogeneity and anisotropy II-Analysis by the single station method, Geophys. J.R. Astron. Soc., 78, 573-617, 1984.

Niazi, M., and H. Kanamori, Source parameters of 1978 Tabas and 1979 Qainat, Iran earthquakes from long-period surface waves, Bull. Seismol. Soc. Amer., 71, 1201-1213,1981.

Wong, Y. K., and J. H. Woodhouse, Ray theory for surface waves on a sphere, Trans. Amer. Geophys. Union, EOS, 64, 260, 1983.

Woodhouse, J. H., and A. M. Dziewonski, Mapping the upper mantle: Three dimensional modelling of earth structure by inversion of seismic waveforms, J. Geophys. Res., 84, 5953-5986, 1984.

Yomogida, K., and K. Aki, Total waveform synthesis of surface waves in laterally heterogeneous earth by Gaussian beam method, Trans. Amer. Geophys. Union, EOS, 65, 1017, 1984.

T. Lay and S.Y. Schwartz, Department of Geological Sciences, The University of Michigan, Ann Arbor, MI 48109.

Received February 14, 1985

Accepted March 7, 1985 